ÉGYPTE monde arabe

\section{Égypte/Monde arabe}

8 | 1991

Perceptions de la centralité de l'Égypte 2

\title{
Une couronne d'herbe
}

\section{Abdou' Goubayr}

Traducteur : Carmella Ciaramella

\section{CpenEdition \\ Journals}

Édition électronique

URL : https://journals.openedition.org/ema/1387

DOI : 10.4000/ema. 1387

ISSN : 2090-7273

Éditeur

CEDEJ - Centre d'études et de documentation économiques juridiques et sociales

Édition imprimée

Date de publication : 31 décembre 1991

Pagination : 183-192

ISSN : 1110-5097

Référence électronique

Abdou' Goubayr, «Une couronne d'herbe », Égypte/Monde arabe [En ligne], 8| 1991, mis en ligne le 08 juillet 2008, consulté le 07 juillet 2022. URL : http://journals.openedition.org/ema/1387 ; DOI : https:// doi.org/10.4000/ema.1387

Ce document a été généré automatiquement le 7 juillet 2022.

Tous droits réservés 


\title{
Une couronne d'herbe
}

\author{
Abdou' Goubayr \\ Traduction : Carmella Ciaramella
}

\section{NOTE DE L'ÉDITEUR}

Texte tiré du recueil Tâg min al-'uchb, éd. Mukhtârat Fusûl, Le Caire, 1985.

1 Je devais partir le matin vers dix heures, je ne sais au juste. Le train serait sans doute en retard, comme d'habitude. Je serais bien parti de bonne heure, mais il n'y avait pas de train plus tôt.

2 Des bruits... Le chant du coq, les ânes, les troupeaux. Des bruits qui filtrent de toutes parts, brisant le silence de la nuit. La lumière par la fenêtre, peu à peu. Puis des pas.

3 - Debout mon gars, viens déjeuner!

4 Il doit être six heures, cinq peut-être. Il faut s'extraire des couvertures, obéir. Que dire, le matin d'un jour comme celui-ci ? Déjà la migraine me martèle la tête. Toute la nuit ces rêves obsédants, quelques brefs sommes, puis ce cauchemar...

5 La helba avec du lait, le thé... Haleine fétide. Où sont mes chaussons? La voix de ma soeur à l'intérieur. Ils vont égorger le pigeon pour moi. Est-ce que ce sera un beau voyage? J'aimerais m'asseoir près de la fenêtre et laisser errer mon regard: les champs, de petites silhouettes au loin, qui cheminent sur une étroite bande de verdure, ou bien, à l'horizon... Ah, des oiseaux.

6 - Tu pars demain? T'as tout préparé ? T'es bien prêt?

7 Mon père, passé me voir hier soir selon son habitude excédante - j'étais dans ma chambre, je triais mes papiers.

- Pourquoi donc qu't'as pas été saluer tout le monde ? Oublie pas.

9 Trois jours que je diffère le moment. Plus moyen d'y échapper. Je vais commencer par ma tante, puis ma grand-mère, la maison de mes oncles. Les voisins. Ils seront tous là pour me dire au revoir et fêter ça. Quelqu'un pleurera, bien sûr. Salwa, sûrement. Elle 
ne savait donc pas que je partirais un jour? Que je ne reviendrais peut-être pas de si tôt ? Que ce n'était qu'un jeu ? Cette gravité terrible, chez elle...

10 - Alors, ton déjeuner? Ça va être froid. Remue-toi donc...

11 Je me suis levé, ai allumé le vieux poste. Psalmodie du Coran. Une voix agréable, ma foi. Où sont mes chaussons? Fatigué de chercher. Mal au dos... Vertige. Et puis, comme d'habitude : les voilà.

12 J'ai ouvert la fenêtre. Une brise humide et froide est entrée. Cela m'a fait du bien. Puis j'ai eu froid, j'ai quitté la fenêtre. Ce serait un rêve que cet endroit si la lumière tombait sur ce haut lit de cuivre, couvert d'une étoffe passée. Mais on dirait un suaire.

Dans la salle de bains, incapable de rien faire d'autre que jouer avec mes doigts dans l'eau de la bassine. Je me suis vaguement aspergé le visage d'eau froide, me suis séché. Je suis retourné au lit, me suis à demi enveloppé dans la couverture pelée. Je me suis mis à siroter la helba, puis j'ai pris du thé, allumé une cigarette.

24 Le temps passe, les bruits affluent.

- Tu vas chercher le fou?...

- Non, le lait, chez Oum Muhammad.

- Bon alors je vais au foul. Hosni! Hé, Hosni!

Ces deux-là chuchotent. Il se passe quelque chose derrière la fenêtre. Les voix se dissipent. La cigarette a un goût de sel dans ma gorge. Les oiseaux sur l'arbre, devant la maison, éclatent en piaillements stridents. Mon père, une fois, a failli en devenir fou. Que faire de moi ? Sortir peut-être...

Voilà mon père qui arrive et me lance ses ordres : «Faut qu' t'ailles chez ta grandemère !» Encore... « Prends ces sous et passe à la boutique. » Sa voix enrouée et lasse. Ses yeux enfoncés dans leurs orbites, qui me scrutent...

J'ai commencé à m'habiller. Pantalon serré à étouffer, chaussettes trouées. La migraine est venue. Quelque chose qui ressemblait à une crise d'épilepsie.

Trois revues qu'il ne faut pas que j'oublie... Je les lirai peut-être l'une après l'autre. Je ne sais pas encore ce que je vais faire dans le train. Peut-être écouter le rythme monotone des machines, ou dévisager mes compagnons de voyage. Est-ce que cette idiote sera en face de moi?

- Bonjour Fulla!

- Tu pars aujourd'hui?

- Oui...

- C'est bien...

- Bien, Fulla?

Qu'a donc son visage las, où il reste des traces de henné ? C'est peut-être seulement pour ce petit animal de soeur que je reviendrai un jour, est-ce que je sais?

D'énormes coups de pioche ébranlent l'espace. Je voudrais m'échapper, me raconter des choses heureuses dans un lieu sans limites, une solitude absolue.

- Tu vas te lancer dans la vie, Abou Zayd... attention!

C'est mon brave oncle Sulayman. Il ne parle pas beaucoup, garde ses secrets pour lui. J'ai le pressentiment qu'il mourra pendant mon absence, je l'ai lu dans ses yeux. 
31 A peine l'idée m'en est-elle venue que... « Je vais me balader au marché », ai-je lancé. Le soleil était bas à l'ouest. Au bout du chemin, des enfants chargés de récipients. Je me suis glissé dans la ruelle, sous les fenêtres, avant qu'elles ne s'ouvrent. Continuer. Aller jusqu'au bout...

32 - On a une nouvelle maîtresse, drôlement belle.

33 - Elle est bête, je connais la géo mieux qu'elle.

34 - Je lui dirai, espèce de mal poli.

35 Ces voix insolentes m'arrivent de derrière. Rien à dire. Je vais tout quitter, tout me quittera, me laissant l'impatience pour seul refuge.

Les boutiques sont encore closes... « Manios Batrios, Epicerie de l'Honnêteté »; "Chez Al-Wahab Salim, épicerie de la Fidélité »; "Cornichons doux... », etc. À force d'avoir lu ces enseignes, je les ai gardées en mémoire... Je les ai lues jusqu'au dégoût. Courir...

Ou ralentir le pas?

Le marché couvert est toujours fermé. Vaste et sombre. Le traverse, frôlant les bâches en loque qui pendent du toit et des portes des boutiques, un vent bavard qui chuchote des histoires de commerce - histoires accablantes, à l'image de la vie... elles réjouissent ceux-ci et affligent ceux-là.

Sur la corniche, des kazurina bientôt en fleurs.

Elle a une histoire étrange, cette ville que j'aurai quittée dans quelques heures.

41 - Pardon, le train est parti depuis combien de temps?

42 Question tant de fois entendue lorsque Kamal, Mamduh, Salah et moi, ou d'autres encore, allions chaque soir déambuler sur la corniche, puis traversions le pont tout en bavardant, cigarette aux lèvres, marchions jusqu'à la gare entre les arbres de la colonie. Nous ne partions pas, allions seulement regarder les étrangères dans les trains qui allaient et venaient. Et rêver - c'était le temps désormais enfui, où l'on rêvait. Il est temps que j'aille frapper chez ma grand-mère.

44 - Bonjour Nabaweya!

45 - Qui est-ce?

46 - Grand-mère est là ?

47 - Ta grand-mère ? Oui, elle est là...

48 Elle est assise sur sa banquette dans la grande pièce aux boiseries hautes, installée sur sa fourrure. L'embout du narghilé entre les mains, elle ne s'aperçoit de ma présence que lorsque je lui touche l'épaule, lève alors les yeux et, surprise :

49 - C'est toi, Abou Zayd?

50 - Grand-mère, je m'en vais.

$51-$ Hein?

52 - Je m'en vais.

53 - Pour travailler?

$54-$ Oui...

55 - J'ai toujours dit à ton père qu'il aille travailler là-bas un bout de temps, mais il ne m'a jamais écoutée. Toi, t'es un brave gars. 
56 Je suis resté avec elle un moment en regardant autour de moi - le tambour accroché au mur, les chapelets et les clés, les pigeons sautillant sur l'échafaudage, la chienne marron. Nabaweya, tapie au coin du feu, remuant les braises pour le narguilé. Le silence qui emplit la maison, murmure comme un vent étouffé.

57 Je suis sorti, dévalant les escaliers comme je le faisais par le passé - quand c'était un plaisir.

Chez ma tante, j'ai saisi le heurtoir, ai frappé la porte de ce rythme allègre que m'avait appris son fils, l'instituteur. Elle est apparue en fredonnant la complainte qu'elle chante à chacune de mes visites, ajoutant cette fois ce vers tronqué : « Tu pars et nous laisses, Tu pars. »

59 - Oui...

60 J'ai senti quelque chose bouger. Ce n'était pas comme ma grand mère, qui était restée assise sur sa fourrure. Quelque chose m'échappait. Elle a mouillé mon visage de ses baisers humides, m'a laissé son odeur.

61 Je me suis dirigé vers la maison des oncles - il fallait gravir des butes de terre battue puis longer les rues étroites et malpropres, tourner, marcher jusqu'à un endroit planté de palmiers. Là, à côté de l'ancienne saqiya, un gamin solitaire, derrière la vache, chantonnait d'une voix plaintive. De temps à autre quelqu'un y menait boire un âne ou une vache, s'en retournait en silence. C'est là qu'était la maison de mes oncles, vaste et isolée.

62 Je me suis assis tant de fois sur ce tronc d'arbre, à côté du grand portail de bois... Ici, c'est avec une pierre que l'on frappe.

63 Le couloir interminable, qui aboutit à la cour où se tiennent les femmes. Mes oncles, de l'autre côté, étendus au milieu du bétail ou triant les graines dans la cour, sous le grand palmier.

64 J'ai souvent essayé d'escalader cet arbre. C'est demeuré un rêve suspendu à son sommet, près des nuages.

65 C'est la femme de l'oncle Kamal, de sa voix faible et cassée, qui m'a répondu. Dès qu'elle m'a aperçu, elle a ramassé son voile, a dit que je ne venais que pour partir. Je l'ai devancée dans le couloir tandis qu'elle marmonnait : « Les tambours résonnent pour les pères et les mères, on psalmodie des versets, on bâti des maisons... »

66 J'ai manqué défaillir.

67 « ... le sang sur les lits, les gémissements des filles, les coups qui résonnent du matin au soir, les nuits, les jours, les ventres gros, qui font mal, on hurle, c'est comme du feu, le sang, la douleur, le grincement du lit, les veilles, le linge à laver et à étendre, les bains... Tout ça et on vous quitte...

Ton oncle est là-bas dans la cour. »

La femme de l'oncle 'Abd Al-Fattâh me croise en chemin, elle se penche, me tend une main râpeuse, me parle en aboiements étouffés, recule lourdement comme une vache pleine.

70 J'écarte le petit échaudage derrière la porte. L'oncle Kamal est là dans son sarwal blanc, maculé, le tamis entre les mains, il trie la paille, accroupi, pioche dans un tas à sa droite, rejette dans un autre à sa gauche. 
71 Au fond de la cour, sous l'auvent comme une ombre frêle, la femme de l'oncle 'Ibâdi se tient à côté de la buflesse, portant quelque chose.

«Salut, toi ! »C'est la voix de Kamal. L'oncle Muhammad charge sur le dos de 'Ibâdi un gros sac de grain. Je m'assieds à côté de Kamal. «Viens du côté du fleuve. Le vent souffle du nord vers le sud, attention aux brins de paille qui voltigent, t'assied pas contre le vent, ce serait une bêtise. "J'aimerais, je ne sais pourquoi, que le vent arrache de l'herbe et la pose sur ma tête, que je l'emporte avec moi pendant le voyage. Une couronne d'herbe.

73 J'ai salué l'oncle Muhammad, qui m'a serré la main en souriant. Je savais qu'il me mettrait en garde contre son frère aîné, occupé à secouer le tamis.

74 - Tu t'en vas et...

75 Reste dans ton coin pendant qu'il fait tourner son moulin à paroles et débite de vieilles histoires qui ont un goût de brique rouge. Regarde donc les cordages suspendus, ou les pattes des bestiaux, ou ces pigeons qui escaladent l'auvent de chaume. Les nids des pigeons et des oiseaux chétifs. Ou les queues des bestiaux qui avancent devant la roue de la saqiya.

L'oncle 'Ibâdi a chassé la paille de ses cheveux tout en secouant sa calotte blanche. Son torse, impressionnant comme une cuirasse cloutée.

« Voilà un jeune homme dangereux... » Il a tendu sa main, a broyé la mienne, comme un cauchemar une main d'enfant.

Les choses vivantes qui m'entourent vont entrer en moi, la plaie va s'élargir encore.

79 La femme de Kamal est arrivée en marmonnant. Elle portait, sur un plat de cuivre poli, quatre tasses et une théière, de cuivre elle aussi. J'ai bu une gorgée, elle a passé le plateau aux hommes, ils ont bu, j'ai siroté le thé en évoquant les jours passés. J'ai salué les hommes un à un. J'avais du mal à garder les yeux ouverts.

80 En partant j'ai croisé les enfants. Je leur ai donné à chacun une piastre, ai serré les petites mains et m'en suis allé. Je suis retourné sur les collines, ai franchi deux ou trois tournants, enfin suis arrivé. Les voisins étaient là, et parmi eux ma mère, un éclair furtif sur le visage.

81 J'avance. Un tremblement dans le genou. Les femmes en noir approchent, m'entourent, odeur d'étoffe poussiéreuse, visages livides, mobiles et toutes ces mains...

82 M'arrêter?

83 Mes grands cousins sont assis dans la pièce. Ils parlent de mon départ eux aussi. Je prend à celui-là sa cigarette, en aspire une longue bouffée puis la lui rends.

84 La carriole est dehors. On charge ma valise et le cageot, où est-ce que je vais ?

85 Je me suis assis sur le cuir souple, eux de chaque côté. Le conducteur a agité les fines rênes, a fait claquer le fouet. Il a tourné, a gravi les collines, est arrivé au marché où l'on a fait halte.

86 Mon père était sur son banc devant la boutique, à ses côtés oncle Salim, tous deux appuyés sur leur canne et regard au sol. J'ai sauté à terre et oncle Salim m'a serré machinalement contre lui. J'ai salué oncle Boutros, le barbier, et ses clients. Enfin mon père. Je suis remonté. Autour de moi, tous ces jeunes garçons, leur odeur de sueur.

87 La corniche. L'eau qui scintille sous le soleil. 

la carriole la gare les voisins. Salwa arrive, jaillit de leur groupe et s'agrippe à moi. Ma mère se fige, ma soeur crie : "Qu'est-ce qui se passe ? » Les femmes, effrayées, reculent. Elle s'agrippe et se penche, se penche. Jusqu'à disparaitre.

100 - Salut! Vous êtes soldat?

101 - Je suis militaire dans la cavalerie. J'ai combattu deux fois.

Les arbres somnolents sur la rive.

La longue route calme.

Le pont en pierre.

Le galop monotone des sabots. L'air chargé de musc.

J'ai allumé une cigarette, mes compagnons m'ont imité. On a traversé les orangeraies éclats jaunes sur fond vert. Vastes espaces. Soleil. Somnolence.

Je me suis éveillé brusquement. J'étais sur le quai de la gare, bâtie à l'anglaise. Le train est arrivé, m'a emporté. J'ai posé mes affaires sur le porte-bagages, me suis installé près de la fenêtre. Les mains ont glissé hors de la vitre. Le quai s'est effacé, les champs lui ont succédé. Grouillement de visages dans le wagon, visages là-bas qui disparaissent tandis qu'il file.

J'ai soupiré, ai allumé une cigarette, rejeté une bouffée. Je me suis mis à observer l'étrange jeune homme à la tête rasée qui me faisait face. Un militaire en civil. Les champs qui s'étendent et disparaissent derrière les montagnes. Des oiseaux comme des gouttes d'eau en chute libre. Défilé d'images qu'entrecoupent les pylônes et les câbles. Là-bas un troupeau sur le pont, et derrière, un homme chevauchant un âne. Une couronne d'herbe sur la tête de mon oncle. L'odeur du crottin. Les cordages accrochés au mur. Les collines de terre battue. Les tournants, la carriole, les chevaux de race. Les visages des femmes, leurs vêtements noirs autour de moi et, plus bas, le visage de ma soeur cadette.

Longs coups sur les tambours, psalmodies, voix des haut-parleurs. Les sommiers. Les cigarettes. La nourriture. Les moutons. Les chèvres sous le palmier. Le silence des champs. Les vaches qui somnolent dans la cour. La femme de mon oncle. Le tamis. Le lit en cuivre. La helba au lait. Les enfants. La jeune maitresse d'école. Le croissant et la lune. La mariée sous la tente de cérémonie. Le henné, les youyous, les danseuses. Les coups de feu, les cris des hommes, le marié. Les mains enlacées, les nouveaux coffres. Le marié soulève le voile, elle détourne son visage de lui. Il la dévêt et lui donne la bourse. Elle rit. Il tend la main vers ses cheveux et en dénoue les longues mèches. Elle se penche. Il se penche et l'embrasse.

Elle ferme les yeux, se dérobe. Il se jette sur elle. Elle gémit. Il glisse le couteau. Elle crie, il sent la présence du sang. Elle tremble. Elle tremble, tremble. Il se redresse. Le mouchoir. Le sang. Le pigeon. Le henné. Le cadeau de mariage. Les plateaux de cha'riyya. Les matins. L'aller, le retour. Le travail. Les vaches. Les moutons. La grossesse. La souffrance. Les jours, les mois. La douleur, les cris. L'enfant. Le travail. Les champs. Les poteaux télégraphiques. Les semences. Les coups.

\section{Toi-même.}

Toi-même toi-même toi-même toi-même toi-même toi-même toi-même toi-même...

Le visage du militaire. Les poteaux télégraphiques les fermes les champs les troupeaux 


\section{ANNEXES}

\section{Interview de Abdou' Goubayr}

Propos recueillis par Carmela Ciaramella dans le cadre d'un mémoire de DEA sur Les «jeunes écrivains » égyptiens, 1960-1970, Aix-en-Provence.

« Je suis né à Esna dans le gouvernorat de Qena, d'une famille appartenant à la classe moyenne. Mes parents sont eux aussi issus de familles de fonctionnaires de province, c'est-à-dire de la petite bourgeoisie rurale égyptienne. Mais on n'est pas automatiquement en accord avec son milieu d'origine...

Mon père et ma mère sont d'Esna, comme mes grands-parents. Si je remonte plus loin, je trouve des ancêtres à Madina, dans la péninsule arabique. Chez mon grand-père, dans le Sa'id, il y a un arbre généalogique, comme dans beaucoup de maisons de HauteÉgypte. Les Sa'îdis sont fiers de leurs origines arabes et se fâchent lorsqu'on les rattache à l'Egypte pharaonique. Ils pourraient tuer pour ça... Je ne suis pas de cet avis mais eux sont comme ça...

Quand j'ai commencé à écrire, c'était sur le Sa'îd, sur les gens d'une petite ville de Haute-Égypte. J'ai commencé à publier des nouvelles en 1967, dans des revues et journaux. Après en avoir publié sept ou huit, j'ai découvert que le Sa'îd constituait un monde à part. J'ai été amené à cette découverte par ma sensibilité et par l'expérience d'autres écrivains, originaires, comme moi, de cette région : Al-'Abnûdî, Yahya Al-Tahir 'Abdallah, Amal Dunqul et Baha' Al-Tahir avaient, avant moi, écrit sur le Sa'îd. On ne peut pas se contenter d'écrire de courts récits sur cet « univers »; pour donner une image complète de ses assises culturelles, de son esprit, il fallait réaliser une oeuvre de vaste envergure. J'ai commencé à rassembler de la matière ; je me suis fait aider en cela par mes quatre sœurs, qui sont institutrices et enregistrent pour moi les histoires que racontent à leurs élèves leurs grands-parents. Je travaille à ce projet depuis 1970 ; il s'agit d'un roman en neuf parties, dont chacune compte 600 à 700 pages. Parallèlement, 
j'écris aussi d'autres choses car j'essaie de travailler mon écriture, de créer une langue, un rythme, un esprit qui me soient propres.

Le roman auquel je me suis attelé évoque une famille de neuf personnes - grandsparents, parents, enfants et petits-enfants - à travers lesquelles je raconte l'histoire de tout le pays, de tout le « Sud » : les problèmes sociaux, les traditions, les souvenirs... [...]

J'ai fait toutes mes études dans des écoles azharistes, des classes primaires jusqu'à l'université. D'abord à Esna puis au Caire, où j'ai étudié la langue et la littérature arabes avant de me spécialiser en littérature anglaise et traduction simultanée. Quand j'étais à Al-Azhar, j'essayais en même temps d'avoir une vie sociale et j'ai toujours pensé que ces études ne me convenaient pas. Aussi les ai-je abandonnées dès que j'ai pu. Le but des études azharistes était de faire de moi un chaykh, mais je pensais depuis mon enfance que je n'étais pas fait pour ça. On peut cependant percevoir, en lisant mes nouvelles, cette formation classique que j'ai reçue contre mon gré. J'ai lu beaucoup de poésie, je connais bien la grammaire et j'ai un vocabulaire très riche. En outre j'ai appris le Coran par coeur quand j'avais neuf ans, ce qui m'a fait pénétrer les secrets de la langue arabe, son rythme, sa beauté. Dans mes livres on retrouve tout cela. Dans la nouvelle Tahriq alQalb, il y a un passage que même les traditionalistes apprécient.... C'est le résultat de ma formation « coranique ». Si l'on considère le Coran du point de vue du rythme, de la syntaxe et des expressions, l'on se rend compte que l'exégèse qu'en font les hommes de religion est réductrice car, contrairement à ce qu'ils croient parfois, le Coran n'est pas seulement un ensemble de règles, il renferme aussi l'esprit du peuple arabe au plus haut degré. Parmi toutes les tribus arabes de l'époque de la Révélation, les Quraych sont ceux qui ont le mieux perfectionné leur langue, c'est grâce à cela qu'ils ont assuré leur suprématie.

Pour en revenir à ma jeunesse et aux différends avec ma famille, j'ai quitté Esna pour échapper à mon destin de chaykh et participer à une vie culturelle qui là-bas n'existait pas. Je suis venu au Caire contre la volonté de mon père et à la suite de cette fugue, mes études à la faculté au Caire ont été une sorte de compromis, jusqu'à ce que je devienne économiquement indépendant. Mes rapports avec mon père, à cette période, étaient assez tendus. Même quand il venait au Caire il ne descendait pas chez moi, à cause de mon comportement «scandaleux » (drogue, femmes, politique...). Ensuite nous avons trouvé un équilibre : quand ma famille a vu que j'écrivais et publiais, que je me « faisais un nom ", elle a commencé à me respecter. Ils restent soupçonneux quant à mes liens avec la religion, mais je suis de plus en plus amené à m'intéresser à leurs problèmes et en particulier à ceux de la dernière génération de la famille. Par exemple j'ai soutenu mes quatre soeurs dans leurs conflits : l'aînée était amoureuse d'un homme qui venait d'une famille pauvre et mon père s'opposait à ce mariage. J'ai pris le parti de ma sœur et elle a fini par épouser l'homme qu'elle avait choisi. Il y aussi les jeunes d'Esna qui, lorsque j'y retourne, viennent me voir, discutent avec moi et j'essaie de faire quelque chose pour eux. J'ai créé à Esna une grande bibliothèque qui porte mon nom et j'organise, chaque fois que j'y vais, des conférences, des rencontres...

Tout ce que j'ai publié jusqu'ici concerne mon expérience d'opposant, de jeune intellectuel dans une société sous-développée. Ce qui me préoccupe essentiellement est de savoir si l'on peut séparer le thème d'une œuvre de sa forme. Je pense que non. En tant que nouveaux écrivains, Sun'allâh Ibrahim, Ibrahim Asian, Yahyâ al-Tahir 'Abdallah et moi faisons un travail qui diffère de celui des générations qui nous ont 
précédés, comme Naguib Mahfouz ou Youssef Idris.... En d'autres termes, nous pensons qu'il n'y a pas de « contenu » (mawdû')en dehors de l'oeuvre. Celle-ci est une entité vivante, complète, dont on ne peut extraire le contenu pour l'en séparer.

À la fin des années 50 , la société égyptienne a vécu l'expérience violente de la révolution du 23 juillet. Cette dernière a abouti à une crise sociale, car elle n'a pas réussi à répondre aux questions vitales qui surgissaient dans les années 40 . Epoque où, déjà, certains écrivains parlaient d'une nouvelle forme artistique. L'on se demandait si l'écrivain était un simple historien ou s'il devait avoir du monde, de la langue, de la technique, des instruments de son art, une vision complète. [...]

Ce qui nous différencie fondamentalement de nos prédécesseurs, c'est notre conception du fait littéraire en général, comme je l'ai expliqué. Par conséquent la production diffère également : Naguib Mahfouz considère la nouvelle comme l'enregistrement d'un accident social dans un moment historique particulier. La crise du logement lui suggère, par exemple, la nouvelle Al Hubb fí hadabat al-haram .... Personnellement, si je devais me pencher sur cette question, je ferais une étude sociologique sur la crise du logement. Mais ce que je veux faire ce n'est pas de la sociologie, c'est de la littérature. Le changement, par rapport aux anciens, est qualitatif et non quantitatif. Actuellement le roman est, à mon sens, la forme littéraire qui peut, mieux que toute autre, traduire ce que nous vivons. Dans l'Antiquité, chez les Bédouins, la poésie remplissait la même fonction. Les représentants de notre courant essaient chacun leur côté de forger leur propre langue, leur propre style. Du moment que notre travail n'est pas centré sur le contenu, l'effort fondamental porte sur la forme. [...] Je suis totalement opposé à une interprétation sociale (tasfír ijtimấî) de la littérature. [...] Je suis d'accord avec Edouard al-Kharrat quand, en sa qualité de critique, il nie le lien mécanique entre questions sociales et littérature. Par ailleurs, il refuse également que l'on applique des idées préconçues à une oeuvre littéraire; mais dans les faits, lui-même a un point de vue particulier, celui d'un copte égyptien qui croit en l'existence d'une " personnalité copte ».

Je ne parle pas - notez le bien - en termes de génération. On pourrait nous considérer comme un courant littéraire qui opte pour une relative indépendance de l'art par rapport aux questions sociales, politiques et économiques de la société égyptienne. Je veux parler d'Aslân, de Sun'allah, d'Al-Kharrât, de Bahâ' al-Tahir, de 'Abd al-Hakim Qasim et, en tout dernier, de quelqu'un comme Gamal al-Ghitâni, que personnellement je ne considère pas comme un écrivain important [...]. Des écrivains comme Al-Ghitâni, ou encore, Magid Tûbya et Yûsuf al-Qa'id, et d'autres encore, qui appartiennent à l'école de Ihsân 'Abd al-Quddûs et de Naguib Mahfouz, produisent de la matière pour les personnes qui veulent étudier les problèmes sociaux. Ils écrivent de belles histoires, mais ne font pas de littérature. Je crois que l'écrivain a une mission dans la société qui est complètement différente de celle de gens comme N. Mahfouz et les écrivains traditionnels. Ma valeur vient de ce que je produis, et pas seulement du fait que je suis écrivain. D'aucuns pensent que tous ceux qui écrivent deviennent automatiquement des personnages sociaux importants, passent à la télévision, à la radio, etc. Nous ne pensons pas que ce soit là la tâche de l'écrivain. Au contraire, son rôle est d'être avec les gens, de vivre une expérience véritable et d'améliorer son travail. Je prends l'exemple de Youssef Idris. Depuis quinze ans il n'écrit pas, mais il reste «écrivain » et écrit des articles sur n'importe quoi, parce qu'il pense que ce n'est pas important d'écrire des romans ou des nouvelles. Dans un numéro spécial de la revue Fusûl ont été 
publiées les interviews d'une trentaine d'écrivains, entre autres Youssef Idris qui a fait des déclarations dangereuses. Il a dit qu'il ne voulait pas être écrivain mais devenir une personnalité importante, et qu'il s'est rendu compte que la littérature pouvait lui donner ce statut! Cela veut dire qu'il ne croit pas en cet art. Il n'a pas cette foi qui donne à l'écrivain une vision du monde à travers l'art. Y. Idris veut, avec ses nouvelles et ses articles, « éclairer » la société. Nous, en revanche, voulons un renversement de tous les concepts, d'une part pour l'homme - afin qu'il soit considéré pour sa valeur propre - d'autre part pour la production humaine c'est-à-dire, en ce qui nous concerne, tout ce qui touche l'art et la littérature, qui doivent être reconnus au sein de la société pour leurs valeurs intrinsèques, indépendamment de toute autre chose. Les gens qui pensent de cette façon sont les plus radicaux : nous sommes toujours opposés au gouvernement, au pouvoir, c'est nous qui allons en prison et n'en sortons que pour y entrer de nouveau. Les autres sont du côté du gouvernement, de l'État. Donc si, dans notre société en voie de développement, l'on entend par « prestige social » le fait d'être présent dans les média, alors ce sont des gens comme Youssef Idris qui sont concernés. Si en revanche on parle sérieusement de littérature et d'art, alors on peut dire que nous prenons part à ce dialogue. Moi-même je participe selon mes possibilités et mon travail s'inscrit dans ce cadre.

Actuellement j'ai envie de partir du Caire pour aller vivre à la campagne, par exemple au Fayyûm. En fait le Caire n'est plus une capitale ni même une ville, elle est devenue un grand village avec tous les problèmes de la campagne et de la ville en même temps. Bien sûr, on ne peut pas aller trop loin parce que c'est dans la capitale qui se trouvent tous les moyens de communication et de culture, et cela pas seulement en Egypte mais dans la majorité des pays du Tiers-Monde. La bourgeoisie et le gouvernement concentrent la présence des instruments culturels dans la capitale et même Alexandrie est tenue à l'écart de la culture. Auparavant la situation était différente : dans les années 50 il était possible de vivre au plus profond du Sa'îd et d'avoir à sa disposition journaux et revues, qui n'étaient qu'au nombre de trois ou quatre et dans lesquels écrivaient Taha Hussein et Al-'Aqqâd. Pour les livres c'était pareil. Il y a toujours à la campagne des amateurs, des jeunes gens qui essayent d'écrire, c'est ce qu'on appelle « la littérature des provinces » (adab al-aqâlim), mais ces gens ne peuvent pas se passer de la ville parce qu'on ne leur en donne pas la possibilité. [...]

Au temps de Nasser, il y avait davantage de possibilités pour les jeunes artistes qui en étaient à leurs débuts. On avait créé des institutions culturelles comme, par exemple, l'Organisme du Théâtre. La même chose existait pour les cinéastes. Il y avait aussi le service du chômage qui distribuait des salaires pendant plusieurs années. Quand Sadate est arrivé au pouvoir, il a vu dans ces organismes les vestiges du socialisme et du communisme et les a éliminés, parce qu'il haïssait la culture et les intellectuels. L'Organisme du cinéma est passé au secteur privé en entraînant la ruine du cinéma égyptien. De même, l'Organisme des écrivains et dessinateurs au chômage est devenu une institution bureaucratique : ses employés travaillent pour les services secrets et les salaires qu'on touche sont dérisoires (en 1987, 150 LE), ils ne permettent pas à un artiste de se consacrer entièrement à son art. Personne ne vit de son art. Moi-même j'ai travaillé dans la presse et l'édition. J'ai travaillé d'abord à Beyrouth puis au Caire dans une maison d'édition progressiste. Ensuite j'ai créé ma propre maison mais l'affaire a échoué et j'y ai laissé tout mon argent. J'ai été aussi correspondant au Caire de plusieurs revues arabes, comme la revue culturelle hebdomadaire Awrâq, publiée à 
Londres. A ce jour je travaille pour la revue Al-Hilâl comme responsable d'une rubrique culturelle, et comme consultant chez le plus important éditeur de Beyrouth, Dâr alTanwîr.

J'ai publié jusqu'à maintenant quatre livres. Le premier recueil, Fâris 'alâ hisân mîn alkhachab, comporte un long récit et cinq histoires brèves. Il est paru au Caire en 1978, chez Dâr thaqâfa al-gadîda. Le second livre est un roman, Tahrîq al-qalb. Il a été publié quatre fois : en 1980 au Caire, chez Al-Markaz al-'arabî li-l-nachr wa-l-tawzî' en 1981 à Beyrouth, chez Dâr al-Tanwîr, en 1985 en Iraq chez Al-Mu'assasa al-'arabiyya al-'âmma ; enfin en 1987, au Maroc, chez Dâr Tubqâl. Le troisième livre est une nouvelle, Thulâthiyyat sabîl al-chakhs. Elle a également été publiée quatre fois : en 1983 à Beyrouth chez Dâr al-Tanwîr ; en 1986 en Iraq ; en 1986 au Maroc chez Dâr Tubqâl ; en 1987 au Caire chez Dâr al-Kalima. Enfin, mon quatrième ouvrage est un recueil de nouvelles, Tag mîn al-'uchb, dans la collection Mukhtarât Fusûl, et a été publié au Caire en 1985.

L'histoire de la parution de ma première nouvelle est assez intéressante. À l'époque il existait en Égypte un quotidien, al-Masâ', qui avait été créé par les socialistes égyptiens et notamment Khâlid Muhyî Al-Dîn. Par tradition, la page culturelle du vendredi était considérée comme très importante, ceci jusqu'à l'ère sadatienne. C'est grâce à cette page que l'on découvrit Salâh Jahîn, 'Abd al-Rahmân al-Abnûdi, Ibrahim Aslân, Gamâl al-Ghitâni... tous ceux qui, aujourd'hui, présentent un intérêt sur la scène culturelle égyptienne. Cette page était préparée par des intellectuels sérieux qui s'intéressaient aux ouvrages de valeur, que leur auteur soit connu ou non et dans tous les domaines de l'art : littérature, critique, arts plastiques.

Je lisais cette page culturelle avec beaucoup d'intérêt. À l'époque, le Café Riche était une sorte de lieu de rendez-vous littéraire, l'on y venait à six heures du soir discuter de ce qu'on avait lu dans cette page le jour même. Une fois - j'avais alors 18 ans et avais écrit trois ou quatre nouvelles - j'ai choisi l'une d'elles et l'ai portée à la rédaction de la page littéraire d'AI-Masâ'. Le responsable, 'Abd al-Fattâh Al-Gamal, était absent et je fus reçu par son remplaçant, un écrivain égyptien du nom de Farûq Munîb (mort récemment). Je lui ai dit que je voulais faire publier ma nouvelle, puis je suis parti. C'était un mercredi. En général on préparait la page un mois avant la parution, mais le vendredi suivant, ma nouvelle était publiée. Le plus étonnant est qu'on avait écrit sur moi une colonne entière... [...]. Ce soir-là, je me suis rendu au Café Riche pour écouter les commentaires. Les habitués, dont Naguib Mahfouz, Yahyâ al-Tâhir 'Abdallah, Amal Dunqul - discutaient de 'Abduh Gubayr en se demandant qui il pouvait bien être. Je me suis présenté. Après cette première parution, environ 15 nouvelles ont été publiées successivement, toujours dans cette page. Voilà comment je suis entré dans la vie littéraire. Je n'ai rien demandé à personne et n'ai pas eu de recommandations.

Ensuite je suis parti au Liban, où j'ai beaucoup publié. À Beyrouth, j'ai travaillé pour la revue Al-Safir, où je préparais les comptes-rendus des livres récemment parus. J'ai toujours pensé que la meilleure manière de lire un livre est d'en faire le compte-rendu par écrit. D'autre part, ayant réfléchi aux moyens de vivre de mon écriture et de continuer à lire, j'ai trouvé que c'était peut-être là une solution.

À mon retour en Égypte, Sun'allâh Ibrahim, qui travaillait auprès de la maison d'édition Dâr al-Thaqâfa al-Gadîda, a voulu créer une collection de « nouvelle littérature » et m'a demandé d'y participer. C'est ainsi que mon premier recueil a été publié en Egypte, en 
1978. L'année d'avant, en 1977, j'ai eu une courte expérience d'édition. J'avais créé une revue, Kutub 'Arabiyya, qui présentait des comptes-rendus de lectures. Mais après deux numéros, comme je n'avais pas de permis d'édition, les autorités m'ont empêché de continuer.

Actuellement je n'ai pas de problèmes pour me faire éditer, au contraire je dois faire face à une demande continue que je ne peux satisfaire, situation qui dure depuis cinq ans car je n'écris pas facilement. [...]

D'autre part, si je n'ai pas rencontré de problèmes pour être publié, c'est que notre " courant littéraire » a suscité la création, par des gens de notre génération, de maisons d'édition, un peu partout dans le monde arabe. À Beyrouth par exemple, il y a trois ou quatre maisons d'édition appartenant à des jeunes de notre génération, qui ont notre façon de penser vis-à-vis de l'indépendance de la littérature et des problèmes sociaux. Quelqu'un comme le responsable de la maison d'édition Dâr al-Tanwîr ne voudrait publier les oeuvres de Naguib Mahfouz à aucun prix, et préfère publier Ibrahim Asian ou un de mes romans. Au Maroc c'est pareil. Il existe un 'courant parallèle au courant officiel, qui a réussi à se donner les moyens de publier ses productions. Ceux qui ont des difficultés de publication sont les écrivains encore jeunes, inexpérimentés...

En ce qui concerne l'édition locale, je citerai en exemple la tentative d'un groupe de jeunes de Qena qui avaient demandé mon aide pour publier une revue littéraire. Je leur ai conseillé de publier plutôt des études sur les histoires traditionnelles que content les vieux et les poètes populaires. Ces jeunes sont pour la plupart instituteurs : comme je l'avais fait avec mes soeurs, ils ont demandé à leurs élèves de remplir des cahiers et ont ainsi réussi à se procurer de la matière. Malheureusement, cette fois encore, la publication a été suspendue... Les autorités considèrent la culture comme quelque chose de dangereux.

Les problèmes du marché du livre doivent être analysés au niveau du monde arabe car sur ce plan, on ne peut pas isoler l'Egypte des autres pays arabes. Je crois que ces problèmes viennent des gouvernements qui s'alignent sur les États-Unis pour isoler l'Égypte, notamment sur le plan culturel, de l'ensemble du monde arabe. Si les moyens de diffusion du livre étaient mis à la disposition des écrivains égyptiens et arabes, ils pourraient facilement vivre de leurs écrits. Dans le temps, il existait des collections économiques publiant chaque mois un volume qui était distribué dans le monde arabe à 100000 ou 150000 exemplaires. Actuellement il existe une collection koweïtienne, 'Alam al-ma'rifa, qui est distribuée en Égypte à 35.000 exemplaires, et dans le reste du monde arabe à environ 100000 exemplaires.

De nos jours chaque pays a ses lois et son système de distribution. En Égypte, Al-Ahrâm et Al-Akhbâr sontles deux seules sociétés de distribution du livre. Quand je leur présente un manuscrit, s'il est jugé peu « sûr ", il sera peut-être accepté mais ne bénéficiera pas d'une bonne publicité ni d'une bonne diffusion. Le problème est donc pour nous de trouver les moyens de parvenir à nos lecteurs et de leur offrir nos créations à un bon prix. Je prends l'exemple de la collection mensuelle que nous publions à Dâr al-Hilâl, Riwayât al-Hilâl. Chacun de nous voit les stocks de son livre, publié dans cette collection, s'épuiser en quelques jours. Les difficultés sont dues d'une part au système d'édition et de distribution et d'autre part à notre statut d'écrivains « non officiels ».

Notre travail littéraire a une grande influence sur les écrivains très jeunes, ceux qui se forment. En matière de critique, au sein de l'université et en dehors, on a beaucoup 
produit. Sur mon travail, on a écrit l'équivalent de cinq ou six fois le volume de ce que j'ai produit. A l'université et, bizarrement, surtout à l'université de Minya, sept thèses de doctorat sont en cours sur nos travaux, et je partage avec Ibrahim Aslân une thèse de magistère. [...]

En ce qui concerne notre importance dans la vie culturelle, au niveau du monde arabe, nous trouvons un bon public dans les nouvelles générations qui ont voyagé dans le monde arabe et hors de celui-ci. En Iraq par exemple, où je suis allé une fois, j'ai rencontré, dans une ville loin de la capitale, des étudiants qui me connaissaient et connaissaient aussi Asian et Al-Ghitâni. Quelle surprise de trouver là-bas de simples étudiants - et non des intellectuels ou des écrivains - qui étaient au courant de notre existence, venaient discuter de notre travail avec nous, nous critiquaient avec des arguments bien pesés. Je pourrais dire la même chose pour le Maroc, le Soudan, la Syrie et les pays du Golfe. Naturellement, notre position politique entre en ligne de compte ; les gens dont on parle sont opposés aux accords de Camp David, à la paix avec Israël, etc. En outre, ils ont la même conception de la littérature et de la culture, et nous sommes « leurs » écrivains.

Déjà, avant 1967, notre courant littéraire critiquait la période nassérienne, dont on se souvient de nos jours comme d'une période de rêve. Il est vrai que Nasser était nationaliste et anti-colonialiste, mais il ne suivait pas une idéologie précise. Il ne se rendait pas compte qu'il avait mis en place une caste militaire dont la position anticulturelle était encore moins acceptable que celle de l'élite bourgeoise d'avant la révolution. Je crois que sans ces mouvements critiques qui se répandirent dans tout le monde arabe contre la « caste militaire-bourgeoise-nationaliste » qui avait dirigé la Révolution du 23 juillet, ni les nouvelles idées ni la nouvelle littérature n'auraient pu s'épanouir et s'affirmer dans la société égyptienne contemporaine [...].

Quant à la relative liberté que l'Égypte connaît actuellement par rapport à l'époque de Sadate, je pense qu'il faut distinguer deux niveaux : d'une part la liberté « extérieure », d'autre part la liberté « intérieure » de l'écrivain, celle qui lui permet de s'exprimer librement et sans contraintes. Le problème c'est que dans le Tiers-monde, on nous inculque dès l'enfance un grand nombre de tabous. L'écrivain d'un pays comme le nôtre mène, chaque fois qu'il écrit, un combat avec lui-même et doit fournir un effort dix fois plus important qu'un écrivain occidental. J'estime que la situation actuelle en Égypte est «meilleure », mais cela ne veut pas dire que ce soit « la meilleure ». Un changement qualitatif serait souhaitable, afin que ceux qui contrôlent les moyens d'édition et de distribution soient du côté de l'écrivain et lui offrent les possibilités de publier. Or aujourd'hui, dans notre pays, la classe au pouvoir est une classe nouvelle aux goûts vulgaires, c'est une «bourgeoisie parasitaire » pour laquelle l'art se résume à Ahmad 'Adawiya et, dans le meilleur des cas, Châdia, Cherihân, et pour le cinéma 'Adil Imâm. J'estime dès lors qu'il n'y a pas eu de vrai changement. Pour moi il y aura un véritable changement lorsqu'on pourra établir un lien authentique avec les masses et leur offrir ce qu'il y a de mieux dans le domaine de l'art. Même les analphabètes peuvent apprendre à apprécier l'art si on les forme en ce sens. Avant la Révolution, il y avait un journal qui s'appelait Al-Siyâsa. En première page, on publiait toujours des poèmes d'Ahmad Chawqî, qui chantait le jour même Um Kalthûm. Ma mère, qui ne sait ni lire ni écrire, apprenait par coeur ces poèmes en langue arabe classique. Cela prouve la justesse de mon idée. Mais, à mon avis, cela ne peut arriver que s'il advient une 
nouvelle révolution ou un mouvement démocratique qui donne aux gens la possibilité de créer leurs propres partis politiques. [...]

Mon public, ce sont les lecteurs qui peuvent acquérir une conscience « globale et artistique » et s'élever au-dessus des ténèbres de l'obscurantisme et de la corruption. Ce ne sont pas seulement des intellectuels de profession. Ils travaillent dans le domaine de la culture, mais on peut trouver aussi des ouvriers, des employés qui sont parvenus à cette conscience, comme j'en ai rencontré beaucoup en prison.

D'autre part, comme on l'a déjà vu, notre public est plus nombreux dans les autres pays arabes qu'en Égypte, si l'on compare la population égyptienne et nos lecteurs avec celle de chacun de ces pays et ceux qui nous lisent. Ceci parce que dans ces pays, le mouvement culturel est beaucoup plus vivant que chez nous. Ils ont, aux postes clés, des hommes nouveaux et plein d'idées, mais surtout des hommes qui ont acquis cette conscience « globale et artistique » dont je parlais plus haut.

Je suis convaincu toutefois que l'on peut faire mieux en Égypte, parce qu'il existe virtuellement un public de lecteurs : on peut le voir aux ventes que réalisent les livres publiés dans des collections bon marché, comme Riwayât al-Hilâl.» [...]

INDEX

Mots-clés : Goubayr (Abdou'), littérature 